\title{
What is the optimal number of researchers for social science research?
}

Jonathan M. Levitt ${ }^{1}$ and Mike Thelwall ${ }^{2}$

${ }^{1}$ J.M.Levitt@wlv.ac.uk and J.Levitt@lboro.ac.uk fpost publication corresponding author] Statistical Cybermetrics Research Group, Faculty of Science and Engineering, University of Wolverhampton, Wulfruna Street, WV1 1LY Wolverhampton (UK)

Tel: +44 2082489050 Fax: +44 1902321478

${ }^{2}$ M.Thelwall@wlv.ac.uk [pre-publication corresponding author]

Statistical Cybermetrics Research Group, Faculty of Science and Engineering, University of Wolverhampton, Wulfruna Street, WV1 1 LY Wolverhampton (UK)

Many studies have found that co-authored research is more highly cited than single author research. This finding is policy relevant as it indicates that encouraging co-authored research will tend to maximise citation impact. Nevertheless, whilst the citation impact of research increase as the number of authors increases in the sciences, the extent to which this occurs in the social sciences is unknown. In response, this study investigates the average citation level of articles with one to four authors published in 1995, 1998, 2001, 2004 and 2007 in nineteen social science disciplines. The results suggest that whilst having at least two authors gives a substantial citation impact advantage in all social science disciplines, additional authors are beneficial in some disciplines but not in others.

Keywords: Scientometrics, collaboration, citation analysis, co-authorship

\section{Introduction}

The encouragement of co-authorship is often a major goal of research policy. Perhaps the

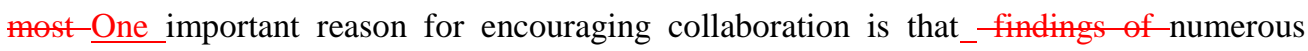
studies (described below) indicate-have found that collaborative research tends to be more highly cited than non-collaborative research. As higher citation is associated with higher research quality, these findings tend to indicate that, in general, collaborative research is of a higher quality than single author research. If policy makers are going to encourage collaborative research, however, it is important to know the optimum number of collaborative partners and the extent to which this varies between disciplines.

Investigations of the relationship between co-authorship and citation have tended to group together all collaborative articles into a single category and to compare the mean 
citation of collaborative articles with the mean citation of single author articles. However, one study has found a found a-significant positive correlation between the number of coauthors and the number of citations (Gazni \& Didegah, 2011) for Harvard University publications and another study found that mean citation within the Science Citation Index (SCI) as a whole increased steadily as the number of authors increased up to at least 12 (Persson, Glanzel \& Danell, 2004).

Gazni and Didegah's findings were for a single institution and Persson, Glanzel and Danell's findings grouped together all SCI disciplines but neither paper investigated entire disciplines, which is a natural level of analysis for policy-relevant findings. To partly fill this gap, this paper investigates the relationship between citations and the number of authors for a selection of social science disciplines.

\section{Background}

Research collaboration leading to co-authored articles can occur in many forms, from $\mathrm{PhD}$ supervision to cooperation between peers (Katz \& Martin, 1997). Although it is a common phenomenon and seems to be widely associated with high productivity, it seems that whilst scientists that collaborate produce more research papers, if fractional counting is used then collaboration does not increase productivity (Lee \& Bozeman, 2005).

The theory of Mode 2 science, an interdisciplinary, applied approach focusing on marshalling all types of expertise necessary to solve an important problem (Gibbons, Limoges, Nowotny, Schwartzman, Scott, \& Trow, 1984; see also: Bordons, Zulueta, Romero, \& Barrigon, 1999; Etzkowitz \& Leydesdorff 2000; Haythornthwaite, 2006), is an influential argument for collaborative research being important for policy-makers to promote. Mode 2 science may be powerful because of its combination of subject expertise and could be more useful to society because of the focus on real-world problem-solving. It may also be more highly cited because of its greater contribution, although applied research can be less cited than pure research (e.g., Seglen, 1997). Although this argument is for the value of interdisciplinary research, most quantitative research has focused on all types of collaboration or the difference between national and international collaboration, presumably because it is difficult to quantify the interdisciplinarity of a set of article authors.

Collaboration varies between countries and disciplines and over time. For example, a comparison between the 50 countries that had at least 1,000 WoS publications in 1995 and 1996, found large national differences: for Switzerland $47.5 \%$ of publications were internationally collaborative, whereas for Japan the figure was only 14.4\% (Glänzel, 2001). A 
regional study also found considerable variation in the collaborative rates of Latin American countries; for 1991-95 the internal collaboration rate was $23-40 \%$ for the five countries that published at least 2,000 papers whereas it was $60-74 \%$ for the five countries that published fewer than 100 papers (Gómez, Fernandez \& Sebastian, 1999).

The rest of this section focuses on published research on collaboration and the relationship between the number of authors and citations. Although working together often results in co-authorship, it does not automatically result in a co-authored publication (Katz \& Martin, 1997; Cronin, Shaw, \& La Barre, 2003; Wang, Wu, Pan, et al., 2005). In addition, although the link between citation and quality is not clear-cut, numerous studies have investigated the relationship between collaboration and citation with a belief that it reflects an underlying relationship between collaboration and research quality.

\subsection{Collaboration and citations}

Within science, many studies have shown that papers with more authors tend to attract more citations. For example, a study using the whole of WoS, which is dominated by the sciences, found a general trend for articles with more authors (at least up to a total of 20) to have higher mean citations (Costas \& van Bochove, 2012). Similarly, a study of a sample of Italian articles from a variety of areas of scholarship found more highly co-authored articles to be both more highly cited and more highly rated by peer reviewers (Franceschet \& Costantini, 2010). A significant positive correlation has also been found between the number of coauthors and the number of citations for Web of Science articles with at least one author affiliated with Harvard University (Gazni \& Didegah, 2011). At the level of individual scientific subjects, each additional author up to 7 increases the average number of citations per article in biomedical research, chemistry and mathematics (Glänzel, 2002). There is a correlation between collaboration and citation for Chinese molecular biology (Ma \& Guan, 2006), but not for well-known molecular biology research institutes (Herbertz, 1995).

Some studies of the social sciences, often using Social Science Citation Index (SSCI) data, have also found statistically significant associations between collaboration in general and citation, including for economics overall, but varying by nation (Levitt \& Thelwall, 2010) and for ecology overall, but not for co-authorships within the same institution (Leimu \& Koricheva, 2005). A sample of 970 Italian economics and statistics articles were also found to be more highly cited and more highly rated by peer review as the number of authors increased from 1 to 2 and from 2 to $3+$ (Franceschet \& Costantini, 2010), although the peer review increase from 2 to $3+$ articles was small $(1 \%)$ and possibly not statistically significant. 
From a slightly different perspective, the proportion of the most highly cited library and information science articles that are collaborative has increased over time but the same is not true for uncited articles (Levitt \& Thewall, 2009). Graduate students on a library and information science course also rated articles more highly if they had more than one author (Finlay, Ni, \& Sugimoto, 2012), suggesting that higher citation may reflect higher quality or utility within this area.

Some studies of the social sciences have not found a relationship between collaboration and-to increase or decrease the average level of citations to articles, however, including investigations of physical education, recreation, and dance (Crase \& Rosato, 1992), finance (Avkiran, 1997), and two library science journals (Hart, 2007).

\subsection{Citations and national or international collaboration}

Several studies of science have found that international articles by more than one author are particularly highly cited. For each of the 31 countries that published the largest number of science papers in 1995, on average, internationally collaborative articles were more highly cited that non-international collaborative articles (Glänzel, 2000). Based upon SCI articles in 1998, the mean citation rate for SCI articles, excluding self-citations, increases at the same rate for national and international articles ( 0.5 citations for each author after the first up to 12) although international collaborative articles received about 1 more citation, on average, than did domestic collaborative articles (Persson, Glänzel \& Danell, 2004). Based on nearly half a million UK SCI publications from 1981 to 1994 (Katz \& Hicks, 1997), articles by authors from two countries on average received about $50 \%$ more citations than articles by authors from a single country. Positive associations between international collaboration and citation rates have also been found for Scandinavian science (Glänzel, 2000), Brazilian science (Leta \& Chaimovich, 2002), New Zealand science (Goldfinch, Dale, \& DeRouen, 2003), and Danish industry (Frederiksen, 2004). In contrast, papers with at least one Harvard University co-author attract less citations the further the authors are apart (Lee, Brownstein, Mills, \& Kohane, 2010), suggesting that international collaboration is ineffective for Harvard authors. This is probably a special case for one elite university collocated with another elite university Massachusetts Institute of Technology) and hence does not undermine the other findings.

At the level of individual subjects, positive associations have been found between citations and international collaboration for Chilean physics (Vogel, 1997), HIV/AIDS in Nigeria (Uthman, 2008) and wood preservative chemical research (Yi, Ao, \& Ho, 2008). An investigation of Spanish Gastroenterology, Cardiovascular Systems and Neuroscience SCI 
articles published between 1991 and 1993 found that international collaboration helped Spanish researchers to publish in higher impact journals (Bordons, Gomez, Fernandez, Zulueta \& Mendez, 1996) and hence, presumably, to attract more citations. Similarly, an investigation of chemistry papers published in 1995 found that for 33 of the 36 countries international collaborative papers were on average more highly cited than non-international collaborative papers (Glänzel \& Schubert, 2001) and a study of nanotechnology articles found that the number of authors was not a significant determinant of citations once other factors, such as various types of internationality, were taken into account (Didegah, \& Thelwall, 2013). These findings demonstrate that, in contrast to international collaboration, national collaboration may not always be associated with higher impact research. An alternative explanation may be that more capable researchers are more able to establish international collaboration or that higher quality research projects are more likely to attract international teams.

One concern when investigating collaboration is that articles by multiple authors seem more likely to be self-cited as there are more authors to self-cite. In partial response, an investigation of astronomy research in the Netherlands (Van Raan, 1998) concluded that higher rates of self-citation in international collaboration do not play a significant role in increasing the citation impact of internationally collaborated articles. A similar result was found for the whole of WoS (Costas \& van Bochove, 2012).

Within the social sciences, links have been found between higher citation and international collaboration for Brazilian management science (Pereira, Fischer, \& Escuder, 2000), library and information science (Levitt \& Thelwall, 2009) and economics (Levitt \& Thelwall, 2010).

A few studies of collaboration have compared more than one country. An investigation of 837 papers published in Oecologia between 1998 and 2000 found a citation advantage for collaborative articles that was higher for US authors than for European authors, although international collaborations were not more highly cited than national collaborations (Leimu \& Koricheva, 2005). Another study generalised this result by showing that the citation advantage gained through international collaboration varied by discipline and country, tending to be greatest in countries with the lowest citation impact and in social sciences and engineering (Lancho-Barrantes, Guerrero Bote, \& de Moya Anegón, 2013). 


\section{Research Questions}

As described in the Background, many studies of single disciplines, particularly within the sciences, have found positive associations between citations and whether an article is collaborative or not and two studies of multidisciplinary or scientific sets of articles have found a correlation between citations and the number of co-authors, up to 7 or 20 . Nevertheless, with the exception of Italian statistics and economics articles with 1, 2 and 3+ authors (Franceschet \& Costantini, 2010) there has been no systematic investigation into the relationship between the number of authors and citation rates within social science disciplines. In order to fill this gap, the relationship between mean citation and number of coauthors is investigated for twenty social science disciplines for five different years, addressing the following research questions:

(1) To what extent does the citation benefit of having more authors vary from discipline to discipline and number of authors in the social sciences?

(2) To what extent does the citation benefit of having more authors vary from year to year in the social sciences?

Although the literature review, albeit mainly for the sciences, suggests that different types of collaboration have different effects on citation rates, and particularly in terms of national, international or institutional collaboration, this study does not differentiate between different types of collaboration in order to get initial wide-ranging findings for the social sciences.

\section{Method}

Data was extracted from the SSCI using the for twenty social science disciplines: (a) the subject area of 'Psychology' and (b) the 19 categories not containing the word 'Psychology' for which over 2,500 SSCI articles were published in 2007. The disciplines with the largest number of articles were investigated, as findings-tests on larger samples have greater statistical power seem likely to beand the results are more widely alid and more-useful. The cut-off of 2,500 articles was chosen because there was a particularly large difference in the number of articles between the discipline with the smallest number of articles over 2,500 (Rehabilitation, 2,514 articles) and the discipline with the next largest number of articles (Anthropology, 2,147 articles). This way of selecting disciplines enabled a comparison of findings for a wide cross-section of disciplines that had a fairly large number of articles.

For each discipline, the mean citation was calculated over all SSCI articles published in the years 1995, 1998, 2001, 2004 and 2007 by one author, two authors, three authors, four authors, and more than four authors. These five years were chosen to cover a substantial 
recent period of time. All citations to articles were included in the analysis (as of early 2013), irrespective of publication date, and so older articles have longer citation windows than younger articles. This study analyses separately articles with fewer than five authors because for some disciplines the number of five author articles was too small for a valid analysis. For example, for the articles published in 2007, three of the disciplines had fewer than twenty 5author articles and a further three had fewer than sixty articles, whereas none of the disciplines had fewer than twenty 4-author articles and only one had fewer than sixty 4author articles. As indicated in the Background the findings are likely to have been similar if self-citations had been excluded.

\section{Results}

Although in all 20 areas there is a tendency for collaborative research to be more highly cited, there are considerable differences between disciplines in the results. For example: (a) The ratio of the citation level for two authors to that for one author for 1995 ranged from 1.06 (Political Science) to 3.98 (Information Science \& Library Science) and for 2007 ranged from 1.16 (Rehabilitation) to 2.59 (Business, Finance), (b) The ratio of the citation level for three authors to that for two authors for 1995 ranged from 0.95 (Economics) to 1.51 (Information Science Library Science) and for 2007 ranged from 0.93 (Law) to 1.45 (Sociology), and (c) The ratio of the citation level for four authors to that for one author for 1995 ranged from 0.77 (Business, Finance) to 4.79 (Information Science \& Library Science) and for 2007 ranged from 1.39 (Rehabilitation) to 43.99 (Psychiatry).

In order to systematically assess the relationship between discipline and number of authors (the first research question), the articles published in the most recent year, 2007, were analysed to see if the number of citations per paper was affected by the number of authors, grouping articles with at least 5 authors together. A Kruskal-Wallis one-way analysis of variance test was used to check for significant evidence of differing distributions of citations for different numbers of authors. 
Table 1: Results of the Kruskal-Wallis test to compare the citation impact of articles published in 2007 with different numbers of authors. In each column, an equals sign indicates no evidence of a difference in citations for articles written by the two stated numbers of authors. An inequality < indicates that articles written by the first number of authors attracted significantly less citations than articles by the second number of authors. Letters are assigned to disciplines with the same set of column values.

\begin{tabular}{|c|c|c|c|c|c|}
\hline Discipline & $\begin{array}{l}1 \text { vs. } 2 \\
\text { authors }\end{array}$ & $\begin{array}{l}2 \text { vs. } 3 \\
\text { authors }\end{array}$ & $\begin{array}{l}3 \text { vs. } 4 \\
\text { authors }\end{array}$ & $\begin{array}{l}4 \text { vs. 5+ } \\
\text { authors }\end{array}$ & Group \\
\hline Psychology & $<$ & $<$ & $<$ & $<$ & A \\
\hline Education & $<$ & $<$ & $=$ & $<$ & $\mathrm{B}$ \\
\hline Environmental & $<$ & $<$ & $=$ & $<$ & $\mathrm{B}$ \\
\hline Psychiatry & $<$ & $<$ & $=$ & $<$ & B \\
\hline Public, Environmental & $<$ & $<$ & $=$ & $<$ & B \\
\hline Social Sciences & $<$ & $<$ & $=$ & $<$ & B \\
\hline Business & $<$ & $<$ & $=$ & $=$ & $\mathrm{C}$ \\
\hline Business Finance & $<$ & $<$ & $=$ & $=$ & $\mathrm{C}$ \\
\hline Economics & $<$ & $<$ & $=$ & $=$ & $\mathrm{C}$ \\
\hline Information Science & $<$ & $<$ & $=$ & $=$ & $\mathrm{C}$ \\
\hline Sociology & $<$ & $<$ & $=$ & $=$ & $\mathrm{C}$ \\
\hline Health care & $<$ & $=$ & $=$ & $<$ & $\mathrm{D}$ \\
\hline Health Policy & $<$ & $=$ & $=$ & $<$ & $\mathrm{D}$ \\
\hline Law & $<$ & $=$ & $=$ & $<$ & $\mathrm{D}$ \\
\hline Neurosciences & $<$ & $=$ & $=$ & $<$ & $\mathrm{D}$ \\
\hline Nursing & $<$ & $=$ & $=$ & $<$ & $\mathrm{D}$ \\
\hline Rehabilitation & $<$ & $=$ & $=$ & $<$ & $\mathrm{D}$ \\
\hline International Relations & $<$ & $=$ & $=$ & $=$ & $\mathrm{E}$ \\
\hline Management & $<$ & $=$ & $=$ & $=$ & $\mathrm{E}$ \\
\hline Political & $<$ & $=$ & $=$ & $=$ & $\mathrm{E}$ \\
\hline
\end{tabular}

The Kruskal-Wallis tests gave statistically significant results for all disciplines $(\mathrm{p}=0.000)$.

Table 1 indicates that for all disciplines, articles written by two authors attracted, on average, more citations than solo articles but there are five different patterns of increasing citations with number of authors. For ease of discussion the following simplification is made: authors 
1-4 are considered to be discrete contributors, but additional contributors produce a manyauthored paper.

- A: Additional discrete authors always increase citations; many-authored papers are the most highly cited.

- B: Additional discrete authors increase citations up to 3 authors; many-authored papers are the most highly cited.

- C: Additional discrete authors increase citations up to 3 authors.

- D: Additional discrete authors increase citations up to 2 authors; many-authored papers are the most highly cited.

- E: A second author increases citations.

Although the results are partly affected by the sample size, so that similar differences may be insignificant in one discipline but significant in another discipline with more articles, some factors are clear from the results. Most importantly, additional authors never significantly reduce the distribution of citations, and for all disciplines a second author significantly increases the distribution of citations. Nevertheless, a third author increases the distribution of citations in about half (11 out of 20) of the disciplines but is only true for a fourth author in one discipline. Hence, outside psychiatry, two or three authors seem to be sufficient for most fields. The exception is for highly co-authored articles with at least 5 contributors, for which over half (12 out of 20) of the disciplines show a citation advantage.

In order to investgate the second reseach question (variation over time), citation levels were calculated for every third year between 1995 and 2007.

For all years and for all disciplines, there is a citation advantage of two author articles compared to single authored articles, although this advantage seems to have reduced between 2001 and 2004 (Table 2). The citation advantage for three author articles compared to two author articles is much smaller, is not universal (exceptions include Economics, Law, Neurosciences, Nursing, Sociology in at least one year), and changes little over time overall, although there is a decrease for most disciplines from 1995 to 2007 (Table 3). 
Table 2: The ratio of citations per article for two author articles to that for single author articles, by year.

\begin{tabular}{|l|l|l|l|l|l|l|}
\hline Discipline & 1995 & 1998 & 2001 & 2004 & 2007 & $\begin{array}{l}\text { Increase } \\
1995-2007\end{array}$ \\
\hline Business & 1.71 & 3.61 & 3.38 & 2.70 & 1.85 & 0.14 \\
\hline Business, Finance & 1.55 & 4.74 & 4.09 & 3.10 & 2.59 & 1.04 \\
\hline Economics & 1.65 & 1.64 & 1.76 & 1.48 & 1.39 & -0.26 \\
\hline Education & 1.90 & 1.71 & 1.63 & 1.64 & 1.74 & -0.16 \\
\hline Environmental & 1.29 & 1.50 & 1.57 & 1.24 & 1.21 & -0.08 \\
\hline Health Care & 1.73 & 1.47 & 1.82 & 1.42 & 1.23 & -0.50 \\
\hline Health Policy & 1.98 & 1.56 & 1.92 & 1.61 & 1.29 & -0.69 \\
\hline Information Science & 3.98 & 1.92 & 2.57 & 2.27 & 2.32 & -1.66 \\
\hline Law & 1.77 & 1.89 & 1.91 & 1.58 & 2.05 & 0.28 \\
\hline Management & 1.19 & 1.68 & 1.45 & 1.59 & 1.25 & 0.06 \\
\hline Neurosciences & 1.76 & 1.84 & 1.28 & 1.01 & 1.44 & -0.32 \\
\hline Nursing & 1.06 & 1.39 & 1.43 & 1.58 & 1.27 & 0.21 \\
\hline Political & 3.74 & 2.40 & 2.54 & 2.13 & 2.24 & -1.50 \\
\hline Psychiatry & 1.89 & 1.71 & 1.57 & 1.56 & 1.45 & -0.44 \\
\hline Psychology & 1.73 & 1.78 & 1.60 & 1.59 & 1.51 & -0.22 \\
\hline Public, Environmental & 1.22 & 1.42 & 1.68 & 1.12 & 1.16 & -0.06 \\
\hline Rehabilitation & 1.50 & 1.50 & 1.36 & 1.22 & 1.56 & 0.06 \\
\hline Social Sciences & 2.12 & 2.21 & 1.96 & 1.75 & 1.57 & -0.55 \\
\hline Sociology & 2.13 & 1.70 & 1.98 & 1.81 & 1.63 & -0.50 \\
\hline Median & 1.73 & 1.71 & 1.76 & 1.59 & 1.51 & -0.22 \\
\hline Table 3: The & & & & \\
\hline
\end{tabular}

Table 3: The ratio of citations per article for three author articles to that for two author articles, by year.

\begin{tabular}{|l|l|l|l|l|l|l|}
\hline Discipline & 1995 & 1998 & 2001 & 2004 & 2007 & $\begin{array}{l}\text { Increase } \\
1995-2007\end{array}$ \\
\hline Business & 1.10 & 1.27 & 1.06 & 1.13 & 1.32 & 0.22 \\
\hline Business, Finance & 1.03 & 1.31 & 1.16 & 1.31 & 1.31 & 0.28 \\
\hline Economics & 0.95 & 1.17 & 1.15 & 1.18 & 1.26 & 0.31 \\
\hline Education & 1.13 & 1.32 & 1.17 & 1.26 & 1.21 & 0.08 \\
\hline Environmental & 1.20 & 1.21 & 1.04 & 1.20 & 1.12 & -0.08 \\
\hline Health Care & 1.29 & 1.21 & 1.32 & 1.14 & 1.14 & -0.15 \\
\hline Health Policy & 1.34 & 1.25 & 1.15 & 1.17 & 1.11 & -0.23 \\
\hline Information Science & 1.51 & 0.84 & 1.07 & 1.31 & 1.11 & -0.40 \\
\hline Law & 0.97 & 0.91 & 1.01 & 1.23 & 0.93 & -0.04 \\
\hline Management & 1.14 & 1.09 & 1.04 & 1.02 & 1.28 & 0.14 \\
\hline Neurosciences & 1.04 & 0.95 & 1.08 & 1.14 & 1.02 & -0.02 \\
\hline Nursing & 1.24 & 1.21 & 1.20 & 0.96 & 1.13 & -0.11 \\
\hline Political & 1.05 & 1.43 & 1.55 & 1.40 & 1.01 & -0.04 \\
\hline Psychiatry & 1.30 & 1.26 & 1.23 & 1.19 & 1.25 & -0.05 \\
\hline Psychology & 1.16 & 1.09 & 1.14 & 1.10 & 1.15 & -0.01 \\
\hline Public, Environmental & 1.13 & 1.10 & 1.04 & 1.20 & 1.10 & -0.03 \\
\hline Rehabilitation & 1.27 & 1.10 & 1.27 & 1.17 & 1.05 & -0.22 \\
\hline Social Sciences & 1.14 & 1.11 & 1.17 & 1.16 & 1.45 & 0.31 \\
\hline Sociology & 1.20 & 1.18 & 1.03 & 0.86 & 1.25 & 0.05 \\
\hline Median & 1.14 & 1.18 & 1.15 & 1.17 & 1.14 & 0.00 \\
\hline
\end{tabular}




\section{Discussion}

A limitation of this study is that it investigates social science disciplines for five years between 1995 and 2007. Although it seems highly likely that the findings would not be much different for other social science disciplines and other recent years, the findings may be very different for much earlier years or for science disciplines. Because the study is on over 300,000 articles, it was not possible to gauge the effect of differences in article quality or breadth of interest on the numbers of citations.

The study is also limited in that it has not differentiated between types of collaboration, such as national and international. It seems possible that disciplines having a larger proportion of international collaboration will be the ones with the highest citation advantage for collaboration. Another important factor is specialism within a research area. It may be that collaborative research within a subject area is more highly cited because it is disproportionately part of a high citation and high collaboration specialist area, such as one involving computing. If this were to be the case then this would make the association between citations and collaboration spurious. It seems unlikely that this occurs systematically in the social sciences, however. The type of collaboration may also affect the results. If two author articles in a discipline are predominantly written by a $\mathrm{PhD}$ student and her supervisor then the high citation of two-author articles may be attributable to $\mathrm{PhD}$ research rather than collaboration per se - and some scientists do not regard co-authorship through $\mathrm{PhD}$ supervision as genuine collaboration (Katz \& Martin, 1997).

From the perspective of the implications of the results, an important limitation is that it is not clear whether co-authored articles require more effort to produce and hence whether co-authored research is an efficient way to generate impact. Finally, the methods here do not demonstrate a cause-and-effect relationship between citations and collaboration: it may be that people conducting highly cited research are more able to collaborate than are others rather than collaboration being a good thing in itself (Weale, 2013).

The use of means based upon skewed data for tables 2 and 3 is another limitation of the paper for part of the results. The general the variability by year for Table 2 is greater than in Table 3, as shown by the values in the last column. For Table 2 the mean range overall is more than three times the mean range for Table 2 than for Table 3 (0.85 compared to 0.27). This difference is due to the mean range across years being highest for 1-author articles (the average across disciplines for 1-author, 2-author and 3-author were 29.8\%, 16.3\% and 19.7\%, 
respectively). The higher percentage for 1-author than 2-author might be because the mean for skewed data can increase with sample size.

\section{Conclusions}

The results suggest that it is a universal social science phenomenon, across time and discipline, that articles with at least two authors are likely to be, on average, more highly cited than articles with one author, and that the difference is likely to be substantial, for example, at least $50 \%$. Nevertheless, unlike the case for SCI articles, additional authors after the second have little effect in some disciplines, although they are advantageous in others.

Based on the findings, policy makers should consider encouraging collaborative research throughout the social sciences, and particularly international collaborative work because other studies have found this to be most beneficial. Nevertheless, policy makers should only encourage larger teams of researchers in some social science disciplines, since larger teams are not universally beneficial. This recommendation is predicated by the assumption that articles take the same total amount of time to prepare, irrespective of the number of authors, and this issue requires further research to verify. Should it be established that collaborative research takes longer total time, for example due to the effort needed to coordinate work, then tables 2 and 3 can be used to help work out how much extra effort would be worthwhile for collaborative research. For example, Business research in 2007 attracted 1.87 times more citations if authored by 2 people than if authored by one person. Hence a collaboration with two authors would be worthwhile if it took less than $87 \%$ extra effort for the collaboration. In contrast, if multi-authored research takes less total time, for example due to efficiencies of specialisation outweighing the time needed for coordination, then collaborative research is always recommended in the social sciences as there seem to be no situations in which it reduces the citation impact of research. Finally, whilst the current findings apply to collaboration in general for the social sciences, future work could differentiate between different types of collaboration (e.g., national vs. international) to get more fine-grained results.

\section{Acknowledgements}

This research was supported by the Economic and Social Research Council [grant reference: RES-000-22-4415]. We thank Gertrude Levitt for her very helpful comments. 


\section{References}

Avkiran, N.K. (1997). Scientific collaboration in finance does not lead to better quality research. Scientometrics, 39(2), 173-184.

Bordons, M., Gomez, I., Fernandez, M.T., Zulueta, M.A., \& Mendez, A. (1996). Local, domestic and international scientific collaboration in biomedical research. Scientometrics, 37(2), 279-295

Bordons, M., Zulueta, M.A., Romero, F., \& Barrigon, S. (1999). Measuring interdisciplinary collaboration within a university: The effects of the Multidisciplinary Research Programme. Scientometrics, 46(3), 383-398.

Costas, R. \& van Bochove, C. (2012). On the Relationship between Author Collaboration and Impact of Scientific Publications. Proceedings of 17th International Conference on Science and Technology Indicators, Montréal: Science-Metrix and OST, 447-448.

Crase, D. \& Rosato, F.D. (1992). Single versus multiple authorship in professional journals. Journal of Physical Education, Recreation and Dancing, 63(7), 28-31.

Didegah, F. \& Thelwall, M. (2013). Determinants of research citation impact in nanoscience and nanotechnology. Journal of the American Society for Information Science and Technology, 64(5), 1055-1064.

Etzkowitz, H. \& L. Leydesdorff. (2000). The dynamics of innovation: from National Systems and "Mode 2" to a Triple Helix of university-industry-government relations. Research Policy, 29, 109-123.

Finlay, S.C., Ni, C., \& Sugimoto, C.R. (2012). New methods for an old debate: Utilizing reader response to investigate the relationship between collaboration and quality in academic journal articles. Library \& Information Science Research, 34(2), 131-137.

Franceschet, M. \& Costantini, A. (2010). The effect of scholar collaboration on impact and quality of academic papers. Journal of Informetrics, 4(4), 540-553.

Frederiksen, L.F. (2004). Disciplinary determinants of bibliometric impact in Danish industrial research: Collaboration and visibility. Scientometrics, 61(2), 253-270.

Gazni, A. \& Didegah, F. (2011). Investigating different types of research collaboration and citation impact: a case study of Harvard University's publications. Scientometrics 87(2), $251-265$.

Gibbons, M., Limoges, C., Nowotny, H., Schwartzman, S., Scott, P., \& Trow, M. (1994). The new production of knowledge: the dynamics of science and research in contemporary societies. London: Sage.

Glänzel, W. (2000). Science in Scandinavia: A bibliometric approach. Scientometrics, 48(2), $121-150$.

Glänzel, W. (2001). National characteristics in international scientific co-authorship. Scientometrics, 51(1), 69-115.

Glänzel, W. (2002). Coauthorship Patterns and Trends in the Sciences (1980-1998): A Bibliometric Study with Implications for Database Indexing and Search Strategies. Library Trends, 50(3), 461-473.

Glanzel, W., \& Schubert, A. (2001). Double effort = double impact? a critical view at international co-authorship in chemistry. Scientometrics, 50(2), 199-214.

Goldfinch, S., Dale, T., \& DeRouen, K. (2003). Science from the periphery: Collaboration, networks and 'periphery effects' in the citation of New Zealand crown research institutes articles, 1995-2000. Scientometrics, 57(3), 321-337.

Gómez, I., Fernandez, M.T. \& Sebastian, J. (1999). Analysis of the structure of international scientific cooperation networks through bibliometric indicators. Scientometrics, 44(3), $441-457$ 
Hart, R.L. (2007). Collaboration and article quality in the literature of academic librarianship. Journal of Academic Librarianship, 33(2), 190-195.

Haythornthwaite, C. (2006). Learning and knowledge networks in interdisciplinary collaborations. Journal of the American Society for Information Science and Technology, 57(8), 1079-1092.

Herbertz, H. (1995). Does it pay to cooperate - a bibliometric case-study in MolecularBiology. Scientometrics 33(1), 1171995.

Katz, J.S. \& Hicks, D. (1997). How much is a collaboration worth? A calibrated bibliometric model. Scientometrics, 40(3), 541-554.

Katz, J.S. \& Martin, B. (1997). What is research collaboration? Research Policy, 26(1), 1-18.

Lancho-Barrantes, B.S., Guerrero Bote, V. P., \& de Moya Anegón, F. (2013). Citation increments between collaborating countries. Scientometrics, 94(3), 817-831.

Lee, K., Brownstein, J., Mills, R., \& Kohane, I., (2010). Does collocation inform the impact of collaboration? PLoS ONE-, 5(12), e14279.

Lee, S. \& Bozeman, B. (2005). The impact of research collaboration on scientific productivity. Social studies of science, 35(5), 673-702.

Leimu, R. \& Koricheva, J. (2005). Does scientific collaboration increase the impact of ecological articles? Bioscience, 55(438), 438-443.

Leta, J., \& Chaimovich, H. (2002). Recognition and international collaboration: The Brazilian case. Scientometrics, 53(3), 325-335.

Levitt J.M. and Thelwall M. (2010). Does the higher citation of collaborative research differ from region to region? A case study of Economics. Scientometrics 85(1), 171-183

Levitt, J.M., \& Thewall, M. (2009). Citation levels and collaboration within library and information science. Journal of the American Society for Information Science and Technology, 60(3), 434-442.

Ma, N. \& Guan, J.C. (2005). An exploratory study on collaboration profiles of Chinese publications in Molecular Biology. Scientometrics, 65(3), 343-355.

Pereira, J. C. R., Fischer, A. L., \& Escuder, M. M. L. (2000). Driving factors of high performance in Brazilian management sciences for the 1981-1995 period. Scientometrics, 49(2), 307-319.

Persson, O., Glanzel, W., \& Danell, R. (2004). Inflationary bibliometric values: The role of scientific collaboration and the need for relative indicators in evaluative studies. Scientometrics, 60(3), 421-432.

Seglen, P.O. (1997). Why the impact factor of journals should not be used for evaluating research. British Medical Journal, 314(7079), 498-502.

Uthman, O.A. (2008). HIV/AIDS in Nigeria: A bibliometric analysis. BMC Infectious Diseases, 8(32) 19 .

Van Raan, A. F. J. (1998). The influence of international collaboration on the impact of research results. Some simple mathematical considerations concerning the role of selfcitations. Scientometrics, 42(3), 423-428.

Vogel, E.E. (1997). Impact factor and international collaboration in Chilean physics: 19871994. Scientometrics, 38(2), 253-263.

Weale, M. (2013). Scientific community: Tapping into success and collaboration, Nature 498, 299. doi:10.1038/498299b

Yi, H., Ao, X.L., \& Ho, Y.S. (2008). Use of citation per publication as an indicator to evaluate pentachlorophenol research. Scientometrics, 75(1), 67-80. 